\title{
Individual participant data meta-analysis provides no evidence of individual response variation in individuals supplementing with beta- alanine.
}

\author{
ESTEVES, G.P., SWINTON, P., SALE, C., JAMES, R., ARTIOLI, G.G., \\ ROSCHEL, H., GUALANO, B., SAUNDERS, B. and DOLAN, E.
}




\section{Individual participant data meta-analysis provides no evidence of individual response variation in individuals supplementing with beta- alanine}

Gabriel Perri Esteves ${ }^{1}$; Paul Swinton ${ }^{2}$; Craig Sale ${ }^{3}$; Ruth James ${ }^{3}$; Guilherme Giannini Artioli $^{1}$; Hamilton Roschel ${ }^{1}$; Bruno Gualano ${ }^{1,4}$; Bryan Saunders ${ }^{1,5}$; Eimear Dolan ${ }^{1}$.

1. Applied Physiology \& Nutrition Research Group; School of Physical Education and Sport; Rheumatology Division; Faculdade de Medicina FMUSP, Universidade de Sao Paulo, Sao Paulo, SP, BR.

2. School of Health Sciences, Robert Gordon University, Aberdeen, UK.

3. Musculoskeletal Physiology Research Group, School of Science and Technology, Nottingham Trent University, Nottingham, UK.

4. Food Research Centre, University of Sao Paulo, Sao Paulo, SP, BR.

5. Institute of Orthopaedics and Traumatology, Faculty of Medicine FMUSP, University of São Paulo, Brazil.

\section{Corresponding author:}

Eimear Dolan:

Faculdade de Medicina FMUSP, Universidade de São Paulo - Av. Dr. Arnaldo, 455, $3^{\circ}$ andar, ZIP code: 01246-903, São Paulo - SP, Brazil; Phone: +55 11 2648-1337; Fax: +55 11 3061-7490; E-mail: eimeardolan@usp.br 


\section{Abstract}

Currently, little is known about the extent of inter-individual variability in response to beta-alanine (BA) supplementation, nor what proportion of said variability can be attributed to external factors, or to the intervention itself (intervention response). To investigate this, individual participant data on the effect of BA supplementation on a high intensity cycling capacity test $\left(\mathrm{CCT}_{110 \%}\right)$ were meta-analysed. Changes in time to exhaustion (TTE) and muscle carnosine (MCarn) were the primary and secondary outcomes. Multi-level distributional Bayesian models were used to estimate the mean and standard deviation of BA and placebo (PLA) group change scores. The relative sizes of group standard deviations were used to infer whether observed variation in change scores were due to intervention or non-intervention related effects. Six eligible studies were identified, and individual data were obtained from four of these. Analyses showed a group effect of BA supplementation on TTE $(7.7[95 \% \mathrm{CrI}: 1.3$ to $14.3 \mathrm{~s}])$ and MCarn (18.1[95\% CrI:14.5 to $\left.\left.21.9 \mathrm{mmol} \cdot \mathrm{kgDM}^{-1}\right]\right)$. A large intervention response variation was identified for MCarn $\left(\sigma_{I R}=5.8\left[95 \% \mathrm{CrI}: 4.2\right.\right.$ to $\left.\left.7.4 \mathrm{mmol} \cdot \mathrm{kgDM}^{-1}\right]\right)$; however, equivalent change score standard deviations were shown for PLA (16.1[95\%CrI:13.0 to $21.3 \mathrm{~s}])$ and BA (15.9[95\%CrI:13.0 to $20.0 \mathrm{~s}]$ conditions, with the probability that standard deviation was greater in PLA being 0.64. In conclusion, the similarity in observed change score standard deviations between groups for TTE indicates the source of variation is common and therefore unrelated to BA supplementation, likely originating instead from external factors, which may include, for example, nutritional intake, sleep patterns or training status.

Keywords: $\beta$-Alanine; performance; exercise capacity; individual response; carnosine; supplement. 


\section{Introduction}

Beta-alanine (BA) supplementation is an established nutritional strategy to improve exercise capacity (Saunders et al., 2017a). This is likely due to its capacity to increase muscle carnosine content (Rezende et al., 2020), which acts as an intracellular buffering agent (Blancquaert et al., 2015; Dolan et al., 2019; Trexler et al., 2015). A recent metaanalysis provided evidence that BA supplementation exerted a positive, albeit small magnitude effect $(d=0.18)$ across a range of exercise protocols, while meta-regression identified that exercise type and duration were influential moderating factors, with BA exerting its greatest influence on exercise capacity-based tests lasting between 30 seconds and 10 minutes $(d=0.49)$ (Saunders et al., 2017a). These results align with plausible physiological mechanisms, given that capacity-based tests of moderate duration are most likely to be limited by metabolic acidosis (Bishop et al., 2009). Despite this evidence of a positive average effect at the group level, substantial variability in performance outcomes was identified between studies. For example, both positive and null effects were reported for the influence of BA on an isometric endurance hold test (Bassinello et al., 2019; Derave et al., 2007; Jones et al., 2017; Sale et al., 2012) or on a high intensity cycling capacity test (the $\mathrm{CCT}_{110 \%}$ ) (Danaher et al., 2014; Hill et al., 2007; Patel et al., 2021; Sale et al., 2011; Saunders et al., 2017b; Yamaguchi et al., 2020). Both these tests should theoretically be amenable to BA supplementation, given that they are exercise capacity tests that induce large $\mathrm{pH}$ perturbations and are within the time-durations most likely to be positively influenced by BA supplementation (Saunders et al., 2017a).

Research investigating BA supplementation has also highlighted large within-study interindividual variability. For example, in addition to reporting that BA moderately improved CCT110\% performance for the group, Saunders et al. (Saunders et al., 2017b) also 
identified a wide range of individual changes, with some participants demonstrating apparently large performance improvements (up to 40 seconds), while others showed none, or even a worsening of performance (up to -20 seconds).

Large inter-individual variability is not limited to BA supplementation studies, but is likely to apply to most health and performance related interventions (Atkinson \& Batterham, 2015). Improved understanding of the factors underpinning this variability could improve study standardization and intervention effectiveness, enabling more targeted recommendations, as well as the opportunity for individualized advice and prescription. Investigation of individual response variation is, however, both methodologically and statistically challenging, as has been described in detail elsewhere (Atkinson et al., 2019; Atkinson \& Batterham, 2015; Bonafiglia et al., 2019; Hecksteden et al., 2015; Senn, 2004; Swinton et al., 2018). Briefly, variance in observed change scores across an intervention generally comprises three sources (Atkinson \& Batterham, 2015; Swinton et al., 2018) including measurement error (which comprises instrumentation noise and biological noise, both of which may cause day to day fluctuations in the observed score, even though the true score remains constant); biological variability (which represents actual change in true score across the intervention period, but which occurs independently of the intervention); and intervention response variation (which represents variation directly attributable to the investigated intervention). Theoretically, variation in the observed change scores of the control group will comprise measurement error and biological variability, whilst variation in the intervention group will comprise all three sources. As such, inclusion of a control group enables estimation of the variation in change scores attributable to the intervention itself. 
Currently, little is known about the extent to which variation in observed changes in response to BA supplementation are directly attributable to the intervention. Accurate estimation of inter-individual variability is, however, likely to require relatively large sample sizes which may not be feasible in single studies due to costs, need for invasive procedures and/or time constraints. Additionally, estimation is particularly challenging in studies of ergogenic aids as expected effects tend to be small (Saunders et al., 2017a), while performance related outcomes can be influenced by substantive measurement error and biological variability. Statistical meta-analysis presents an approach to mitigate the limitations of small effects, noisy measurement outcomes and small sample sizes by pooling data across studies to better estimate parameters of interest (Page et al., 2020). Most meta-analyses are, however, based upon aggregate data and can only provide information on the mean response. In contrast, individual participant data (IPD) metaanalyses which source raw data from previous studies, have been described as the "goldstandard" of meta-analytic approaches because they allow for assessment of participant level effects and interactions (Kelley \& Kelley, 2019). Accordingly, we conducted an IPD meta-analysis, the aim of which was to estimate the mean response to BA supplementation on high intensity cycling capacity and to quantify individual response variation (namely that attributable to the BA intervention itself).

\section{Methods}

This meta-analysis was conducted in accordance with the Preferred Reporting Items for Systematic Reviews and Meta-Analysis (PRISMA) guidelines (Moher et al., 2009). Eligibility criteria were defined according to the Population, Intervention, Comparator, Outcomes and Study Design (PICOS), as described in Table 1. Only studies that used the $\mathrm{CCT}_{110 \%}$-a high-intensity cycling capacity test in which individuals are required to 
cycle at $110 \%$ of their previously stablished maximal power output ( $W_{\max }$ ) until volitional exhaustion-were included. This test was selected as a model for this analysis because it is a widely used protocol in which a positive response to BA supplementation would be expected (Saunders et al., 2017a).

\section{Search strategy and data extraction}

To identify eligible studies, the included studies from a previous meta-analysis of all double-blind placebo-controlled trials that investigated the influence of BA supplementation on exercise test performance were screened (Saunders et al., 2017a). The full search strategy is described in the previous meta-analysis. Three databases (PubMed, Google Scholar and Web of Science) were searched using the terms " $\beta$-alanine OR betaalanine" concatenated with "supplementation OR exercise OR training OR athlete OR performance OR carnosine" and only double-blinded, placebo-controlled trials were selected. The same strategies were repeated in September 2020 to identify any additional studies that had been published in the interim. Aggregate data were extracted from studies that matched our inclusion criteria (Table 1). Authors were contacted to request individual participant data, and these were compiled into a pre-piloted excel spreadsheet.

\section{Risk of bias and certainty in cumulative outcomes:}

Certainty in systematic review outcomes (namely each combined meta-analytic result based upon all available data sets) was ascertained using the recommendations of the Grading of Recommendations, Assessment, Development and Evaluations (GRADE) working group (Guyatt et al., 2008). Five potential downgrading factors were considered, namely risk of bias (assessed using the Cochrane Collaborations RoB2 tool (Sterne et al., 2019)), indirectness, inconsistency, imprecision and publication bias. Potential upgrading 
factors included large effects, evidence of a dose-response or the presence of plausible residual confounding factors (Guyatt et al., 2008). All studies in the current review were initially defined as "high certainty" given that only double-blinded, randomized, placebocontrolled designs were included in the review. Application of the GRADE strategy subsequently allowed for this a-priori certainty rating of "high" to be maintained, or to be downgraded to "moderate", "low" or "very low".

\section{Statistical analysis}

The present study comprised both aggregate and individual participant data meta-analyses from a Bayesian perspective. Analyses were performed on change in TTE performance (s) as the primary outcome, and change in MCarn $\left(\mathrm{mmol} \cdot \mathrm{kgDM}^{-1}\right)$ as the secondary outcome. For aggregate analyses, placebo controlled mean difference and variance difference effect sizes were calculated to describe the effects of supplementation on mean response and response variation, respectively. Calculated values were pooled using threelevel hierarchical models including random effects to account for within studies variation, between studies variation $(\tau)$ and covariance $(I C C)$ of multiple outcomes reported in the same study. Standard errors for mean differences (Morris \& DeShon, 2002) and variance differences (Williamson et al. 2018) were calculated according to previously described formulas. The required pre-post correlation for mean difference standard errors was estimated from available data and set to 0.5 for both outcome variables. Response variation estimates were transformed by taking the square root $\left(\sigma_{I R}\right)$ of the absolute variance values and then reapplying the positive or negative sign (Williamson et al. 2018). Individual participant data meta-analyses were conducted by calculating observed change scores from baseline and fitting hierarchical distributional models enabling both the location (mean) and spread (standard deviation) to be estimated assuming Gaussian 
distributions. Models for both the mean and standard deviation included a group factor (placebo vs intervention) and for the mean a participant random effect intercept was added. Moderator analyses investigating group level characteristics were completed for total BA consumption in both outcome variables. However, moderator analyses for individual variation in the primary outcome (TTE performance change) were only conducted where evidence was obtained that variation was influenced directly by the intervention (Atkinson \& Batterham, 2015). Inferences from all analyses were performed on posterior samples generated using the Hamiltonian Markov Chain Monte Carlo method (five chains, 100,000 iterations and 50,000 warmup). Interpretations were based on the median value (0.5-quantile) and credible interval $(\mathrm{CrI})$. Additionally, posterior samples were used to estimate the proportion of individuals that would observe a small, medium and large change (small: 0.2; medium: 0.5; and large: 0.8 times baseline standard deviation), and the probability that the intervention change score standard deviation was larger than placebo for individual participant data analyses. Analyses were performed using the R wrapper package brms interfaced with Stan to perform sampling (Bürkner, 2017). Convergence of parameter estimates was obtained for all models with GelmanRubin R-hat values below 1.1 (Gelman et al., 2013). 


\section{Results}

Overview of available studies

Six studies that investigated the influence of BA supplementation on performance in the $\mathrm{CCT}_{110 \%}$ were identified (Danaher et al., 2014; Hill et al., 2007; Patel et al., 2021; Sale et al., 2011; Saunders et al., 2017b; Yamaguchi et al., 2020). All corresponding authors were contacted, and individual participant data from four studies were obtained (Patel et al., 2021; Sale et al., 2011; Saunders et al., 2017b; Yamaguchi et al., 2020). A summary of the study design, population and dosing protocol of all included studies is available in Table 2. The $\mathrm{CCT}_{110 \%}$ protocols employed were compared to the standard protocol described in a reliability study of the $\mathrm{CCT}_{110 \%}$ (Saunders et al., 2013) (see Supplementary File 1). The differences between study designs were minor, and deemed unlikely to impact interpretation of the results, particularly given that each study contained its own placebo-controlled comparison group who underwent an identical protocol to the BA group.

Influence of BA supplementation on Time to Exhaustion in the $C C T_{110 \%}$ (primary outcome)

Aggregate data for mean difference effect sizes were obtained for five studies (Hill et al., 2007; Patel et al., 2021; Sale et al., 2011; Saunders et al., 2017b; Yamaguchi et al., 2020) generating a total of 11 placebo-controlled effect sizes. One study was not included (Danaher et al., 2014) because no pre-supplementation data for TTE was available. This study did, however, report a positive influence of BA on this outcome when compared to placebo $(p=0.005)$ (Danaher et al., 2014). Using the aggregate data, a large absolute mean difference effect size was estimated $\left(E S_{0.5}=11.9\right.$ [95\%CrI: 6.3 to $16.5 \mathrm{~s}$ ]; Figure 1 A) with relatively low between study variance $\left(\tau_{0.5}=2.4\right.$ [75\%CrI: 0.5 to $\left.6.1 \mathrm{~s}\right]$ and 
covariance due to reporting of multiple timepoints $\left(I C C_{0.5}=0.13[75 \% \mathrm{CrI}: 0.02\right.$ to 0.36]). Evidence of a group level moderating effect was identified for total BA consumption. The mean difference between groups in time to exhaustion with a cumulative dose of $500 \mathrm{~g}$ was estimated to be 7.4 [ $95 \% \mathrm{CrI}$ : 0.8 to $13.9 \mathrm{~s}$ ], with linear regression estimating a 0.6 [95\%CrI: 0.03 to $1.2 \mathrm{~s}]$ ) increase per additional $100 \mathrm{~g}$ consumed. Aggregate data for response variation were calculated for four studies (Patel et al., 2021; Sale et al., 2011; Saunders et al., 2017b; Yamaguchi et al., 2020) generating a total of 9 effect sizes. Large credible intervals were obtained indicating equivalence or potentially greater variation in the placebo group $\left(\sigma_{I R}=-6.1\right.$ [95\% CrI: -15.5 to $\left.11.7 \mathrm{~s}\right]$; $\tau_{0.5}=10.4$ [75\%CrI: 5.1 to $\left.15.3 \mathrm{~s}\right] ; I_{0.5}=0.12$ [75\%CrI: 0.01 to 0.30$\left.]\right)$.

Individual participant data were obtained for four studies (Patel et al., 2021; Sale et al., 2011; Saunders et al., 2017b; Yamaguchi et al., 2020) generating 210 sets of pre, intermediate and post-test data (127 BA, 83 placebo) across 78 participants (46 BA, 32 placebo). Using the distributional model, the mean difference in time to exhaustion with BA compared to placebo was estimated to be 7.7 [95\% CrI: 1.3 to $14.3 \mathrm{~s}$ ] (Figure $1 \mathrm{~B}$ ), with very large individual random effect intercepts estimated (14.1 [95\% CrI: 10.6 to 16.8 s]). In agreement with the aggregate analysis, the distributional model estimated equivalent standard deviation of change scores in the combined placebo (16.1 [95\% CrI: 13.0 to $21.3 \mathrm{~s}])$ and supplement $(15.9$ [95\% CrI: 13.0 to $20.0 \mathrm{~s}$ ] analysis, with the probability that the standard deviation was greater in the placebo group being 0.643 . Using the distributional model estimates, the proportion of individuals expected to make at least a small, medium, and large improvement were substantively greater for BA supplementation (small: 0.63 [95\%CrI: 0.42 to 0.81 ]; medium: 0.52 [95\% $\mathrm{CrI}: 0.31$ to 0.72]; and large: 0.42 [95\% CrI: 0.22 to 0.62$]$ ) compared to placebo (small: 0.45 [95\% CrI: 
0.24 to 0.66 ]; medium: 0.35 [95\%CrI: 0.17 to 0.56 ]; and large: 0.26 [95\% CrI: 0.11 to 0.45]). No moderator analyses for individual variation were investigated due to the similarity in change score standard deviations (Williamson et al., 2018).

\section{Influence of BA supplementation on MCarn (secondary outcome)}

MCarn aggregate data were obtained for four studies (Danaher et al., 2014; Hill et al., 2007; Saunders et al., 2017b; Yamaguchi et al., 2020) generating a total of 10 placebocontrolled effect sizes. A large absolute mean difference effect size was identified $E S_{0.5}=13.7\left[95 \% \mathrm{CrI}: 7.7\right.$ to $\left.19.6 \mathrm{mmol} \cdot \mathrm{kgDM}^{-1}\right]$ with moderate between study variance $\left(\tau_{0.5}=4.2\left[75 \% \mathrm{CrI}: 0.3\right.\right.$ to $\left.\left.12.0 \mathrm{mmol} \cdot \mathrm{kgDM}^{-1}\right]\right)$ and substantive covariance due to the reporting of multiple timepoints $\left(I C C_{0.5}=0.55\right.$ [75\%CrI: 0.23 to 0.82$\left.]\right)$. Evidence of a group level moderating effect was identified for total BA consumption. The mean difference in MCarn with a cumulative dose of $500 \mathrm{~g}$ was estimated to be 15.1 [95\% CrI: 10.7 to $19.5 \mathrm{mmol} \cdot \mathrm{kgDM}^{-1}$ ], with linear regression estimating a 0.36 [95\% $\mathrm{CrI}: 0.09$ to $\left.0.6 \mathrm{mmol} \cdot \mathrm{kgDM}^{-1}\right]$ ) increase per additional $100 \mathrm{~g}$. Aggregate data for response variation were calculated for two studies (Saunders et al., 2017b; Yamaguchi et al., 2020) generating a total of 7 effect sizes. Evidence was obtained indicating a large response variation $\left(\sigma_{I R}=5.8[95 \% \mathrm{CrI}:-2.4\right.$ to $8.4 \mathrm{~s}] ; \tau_{0.5}=3.9[75 \% \mathrm{CrI}: 1.9$ to $6.5 \mathrm{~s}] ; I C C_{0.5}=$ 0.18 [75\%CrI: 0.01 to 0.38$])$

Individual participant data on the MCarn response to supplementation were obtained for two studies (Saunders et al., 2017b; Yamaguchi et al., 2020), generating 156 sets of prepost data (101 BA, 55 placebo) across 39 individuals (26 BA, 13 placebo). The mean difference in MCarn in the supplement condition compared to placebo was estimated to be 18.1 [ $95 \% \mathrm{CrI}: 14.5$ to $21.9 \mathrm{mmol} \cdot \mathrm{kgDM}^{-1}$ ], with individual random effect intercepts 
estimated as 4.3 [ $95 \% \mathrm{CrI}: 3.0$ to $\left.6.0 \mathrm{mmol} \cdot \mathrm{kgDM}^{-1}\right]$. When pooling data across studies using the distributional model, the intervention response standard deviation was estimated as $5.8\left(95 \% \mathrm{CrI}: 4.2\right.$ to $\left.7.4 \mathrm{mmol} \cdot \mathrm{kgDM}^{-1}\right)$.

\section{Certainty in outcomes}

All outcomes were assigned an a-priori certainty rating of "high" because they were all based upon data from double-blinded, randomized, placebo-controlled trials (as defined by the eligibility criteria). Results of the RoB2 assessment are summarised in Figure 2 (generated using R package robvis (McGuinness \& Higgins, 2021)). Possible sources of bias included a lack of information on specific randomization and concealment approaches (Domain 1) (Danaher et al., 2014; Hill et al., 2007; Sale et al., 2011); nonreporting of adherence or compliance information (Domain 2) (Danaher et al., 2014; Hill et al., 2007); and lack of information about the extent of, or reasons for, participant withdrawal (Domain 3) (Danaher et al., 2014; Hill et al., 2007; Sale et al., 2011; Saunders et al., 2017b). Additionally, no study provided a preregistered protocol or analysis plan (Domain 5). These issues were largely due to a lack of detail in reporting, and were deemed unlikely to meaningfully bias the available data. As such, certainty in outcomes were not downgraded due to RoB2 (The complete analysis and decision rationale is available in Supplementary File 2). The studies in this review used commonly recommended dosing protocols, and were conducted on young, healthy, recreationally active but non-specifically trained men and so were not downgraded based upon indirectness, but downgrading of certainty in these outcomes may be advisable for investigators interested in other populations, e.g., highly trained athletes. All MCarn outcomes, and the influence of total BA consumption on TTE performance were downgraded due to potential imprecision as they were based upon a subset of the available 
data. MCarn outcomes were subsequently upgraded, however, because they were consistent with recent meta-analytic results based upon all available data related to the MCarn response to BA supplementation (Rezende et al., 2020), thus leading to a greater level of certainty in the results obtained. Overall, the majority of outcomes were deemed to have a "High" degree of certainty, with the exception of the influence of total BA consumption on TTE, which was deemed to be of "Moderate" certainty (Table 3).

\section{Discussion}

The principal finding of this investigation was the lack of intervention response variation to BA supplementation on high-intensity cycling performance. Large, but similar, variances in individual change scores were observed for both the BA and placebo groups, indicating that variation in response to the intervention itself had a negligible contribution, meaning that observed changes were mainly attributable to factors common to both groups, namely measurement error (instrument and biological noise) and/or biological variability.

The finding of a positive effect of BA supplementation on MCarn aligns with previous research (Harris et al., 2006; Rezende et al., 2020) and confirms that the biological mechanism for improved performance was present. Comparisons between BA and placebo groups identified a large intervention response standard deviation for MCarn changes $\left(\sigma_{I R}=5.8\left(95 \% \mathrm{CrI}: 4.2\right.\right.$ to $\left.7.4 \mathrm{mmol} \cdot \mathrm{kgDM}^{-1}\right)$, demonstrating that a substantial proportion of observed variation was attributable to the intervention itself, which may relate to factors involved in the biokinetics of MCarn synthesis, such as the rate of BA uptake to the skeletal muscle, or the activity level of the synthesizing enzyme carnosine synthase, or in intervention adherence. Previous research also indicates that BA 
supplementation has a large effect on MCarn (Rezende et al., 2020), and that in the absence of intervention, MCarn remains relatively stable across similar time periods as were investigated herein (Baguet et al., 2009; da Eira Silva et al., 2020; Rezende et al., 2020). Both factors (large effect and small biological variation) may facilitate identification of intervention response variation. In contrast, whilst large variations in observed change scores were also observed for TTE, comparisons between BA and placebo groups revealed similar standard deviations. This finding indicates that intervention response variation is negligible, and that observed variation must be primarily attributable to factors common to both the placebo and BA group (namely measurement error and biological variability). These differences in individual response findings for MCarn and TTE highlight the importance of not conflating mechanistic and performance outcomes - just because the biological mechanism to improve exercise performance is present, does not necessarily mean that all individuals will experience an associated magnitude dependent performance improvement.

The finding of a group effect for BA supplementation on performance on this highintensity capacity test, along with negligible individual response variation, has positive implications for individuals who supplement with BA, as it suggests there is a consistent group effect and that most individuals who supplement will have the capacity to improve performance. This does not mean, however, that everyone who supplements will record improvements in performance. As evidenced by the large inter-individual variation observed (see Figure 1), large measurement errors and true changes (both positive and negative) in performance can be caused by sources external to the supplement. Although beyond the scope of the current investigation to parse out the relative contribution of potential factors underpinning this variation, it seems unlikely that the instrumentation 
noise component of measurement error would noticeably impact performance. As such, biological noise (i.e., biological factors that cause the observed score to fluctuate even though the true score remains stable) and biological variability (actual change in the true score due to factors outside the intervention) are the most likely explanations for this finding. Similar findings of negligible variation attributable to the intervention itself has been reported in other interventions (Islam \& Gurd, 2020), including the effect of exercise training on $\mathrm{VO}_{2 \max }$ (Williamson et al., 2017) and weight loss (Williamson et al., 2018), in a pain management intervention in adults with chronic musculoskeletal pain (Watson et al., 2021), and in changes in muscle size and strength following resistance training (Dankel et al., 2020). Collectively, these findings support consideration of more holistic approaches to intervention delivery.

Factors that may potentially influence an individual's response to intervention include nutritional status (both acute and chronic), physical activity levels, sleep, environmental conditions and external sources of motivation (Mann et al., 2014), such as intervention expectancy (Marticorena et al., 2021). A logical next step for future research would be to attempt to parse out the relative influence of these factors on individual response variation, although this is undoubtedly challenging. Initially, parameters of interest must be defined. For example, although it seems logical to predict that factors such as nutritional intake may contribute to observed variability, the precise parameters required to test this hypothesis remains to be determined (e.g., macronutrient composition? Micronutrient adequacy? Energy availability?). It is also important to consider whether parameters of interest can be measured with a reasonable level of accuracy. Further complicating these assessments is the possibility that a combination of factors, exerting potentially opposing directional effects, is likely to underpin observed variation, in which 
case very large samples, in combination with sophisticated analysis techniques, may be necessary to parse out their relative contribution. Sampling error can lead to different outcomes between studies, and this is particularly relevant when investigating small effects and large variability (as is common in sport supplement interventions) and therefore results from any one study should be interpreted with caution. Strategies to increase sample size and thereby to reduce sampling error (for example through multicentre studies or individual participant data meta-analyses as was conducted herein) may be required to further advance understanding of factors underpinning individual variation. Similarly, reducing measurement error through selection of the most reliable tests, rigorous control and standardization of potential confounding variables, as have been described elsewhere (Betts et al., 2020; Burke \& Peeling, 2018), along with increased testing may also facilitate further investigation of individual response variation.

The findings of the present study may have been influenced by the combination of relatively small mean intervention response alongside large measurement error. In contrast, studies investigating interventions with larger mean responses and lower measurement error may have greater precision to quantify the different error sources and potentially identify intervention response variation. It would also be interesting to investigate whether population characteristics impact findings. The participants investigated herein were all young, healthy, active men, but they were not trained cyclists. Replication of these analyses in elite athletes, who may, theoretically, be less subject to both measurement error and external sources of variation (by having more consistent sleep, nutrition and training habits), may potentially allow for detection of variation attributable to the intervention itself. 


\section{Summary and Conclusion}

In both aggregate and individual participant data meta-analyses we identified a positive mean effect of BA supplementation on high-intensity cycling capacity as determined by the CCT110\% test, although there was considerable inter-individual variability in the observed change. The extent of this variation was similar between the placebo and BA groups, indicating that it was mainly due to factors common to both groups and with minimal contribution attributable to the BA intervention itself. Individuals who wish to supplement with BA should follow evidence-based dosing protocols (e.g., to ingest $3.2-$ $6.4 \mathrm{~g} \cdot \mathrm{day}^{-1}$ of BA for at least 4 weeks). In addition to following dosing recommendations, each individual should consider other modifiable lifestyle factors in order to enhance their own likelihood of a positive response, which includes, for example, maintaining dietary habits that support energy and nutrient requirements, recommended physical activity levels and adequate sleep schedules.

Acknowledgements: The authors would like to thank researchers and voluntaries that conducted or participated in the included studies of this meta-analysis. The Risk of Bias figure was created using R package robvis.

\section{Authorship}

Conceptualization, E.D. and P.S.; Methodology, E.D. and P.S.; Formal analysis, P.S.; Investigation, G.P.E. and E.D.; Data Curation, G.P.E. and E.D.; Writing - Original Draft, G.P.E. and E.D.; Writing - Review \& Editing, G.P.E., P.S., C.S., R.M.J., G.G.A., H.R., B.G., B.S., E.D. All authors approved the final version of the manuscript.

\section{Conflict of interest}


Our research group has previously received financial support, supplements free of charge and support for open access publication charges from Natural Alternatives International (NAI, a company that produces BA) for studies unrelated to this one. NAI have not had any input (financial, intellectual, or otherwise) to the present investigation. The authors have no other potential conflicts of interest to declare.

\section{Funding sources}

G.P.E., B.S., B.G., G.G.A. and E.D. are supported by research grants from the São Paulo Research Foundation (FAPESP grants \#2020/07860-9, 2016/50438-0, 2017/12511-0, 2019/05616-6, 2019/25032-9, 2019/26899-6). B.S. has also received a grant from Faculdade de Medicina da Universidade de São Paulo (2020.1.362.5.2).

\section{References}

Atkinson, G., \& Batterham, A. (2015). True and false interindividual differences in the physiological response to an intervention. Experimental Physiology, 100(6), 577588.

Atkinson, G., Williamson, P., \& Batterham, A. (2019). Issues in the determination of "responders" and "non-responders" in physiological research. Experimental Physiology, 104(8).

Baguet, A., Reyngoudt, H., Pottier, A., Everaert, I., Callens, S., Achten, E., \& Derave, W. (2009). Carnosine loading and washout in human skeletal muscles. Journal of Applied Physiology, 106(3), 837-842.

Bassinello, D., de Salles Painelli, V., Dolan, E., Lixandrão, M., Cajueiro, M., de Capitani, M., Saunders, B., Sale, C., Artioli, G. G., Gualano, B., \& Roschel, H. 
(2019). Beta-alanine supplementation improves isometric, but not isotonic or isokinetic strength endurance in recreationally strength-trained young men. Amino Acids, 51(1), 27-37.

Betts, J. A., Gonzalez, J. T., Burke, L. M., Close, G. L., Garthe, I., James, L. J., Jeukendrup, A. E., Morton, J. P., Nieman, D. C., Peeling, P., Phillips, S. M., Stellingwerff, T., van Loon, L. J. C., Williams, C., Woolf, K., Maughan, R., \& Atkinson, G. (2020). PRESENT 2020: Text Expanding on the Checklist for Proper Reporting of Evidence in Sport and Exercise Nutrition Trials. International Journal of Sport Nutrition and Exercise Metabolism, 30(1), 2-13.

Bishop, D., Edge, J., Mendez-Villanueva, A., Thomas, C., \& Schneiker, K. (2009). High-intensity exercise decreases muscle buffer capacity via a decrease in protein buffering in human skeletal muscle. Pflugers Archiv European Journal of Physiology, 458(5), 929-936.

Blancquaert, L., Everaert, I., \& Derave, W. (2015). Beta-alanine supplementation, muscle carnosine and exercise performance. Current Opinion in Clinical Nutrition and Metabolic Care, 18, 63-70.

Bonafiglia, J., Brennan, A., Ross, R., \& Gurd, B. (2019). An appraisal of the SD IR as an estimate of true individual differences in training responsiveness in parallel-arm exercise randomized controlled trials. Physiological Reports, 7(14), 14163.

Burke, L. M., \& Peeling, P. (2018). Methodologies for Investigating Performance Changes With Supplement Use. International Journal of Sport Nutrition and Exercise Metabolism, 28(2), 159-169.

Bürkner, P.-C. (2017). brms : An R Package for Bayesian Multilevel Models Using Stan. Journal of Statistical Software, 80(1). 
da Eira Silva, V., de Salles Painelli, V., Katsuyuki Shinjo, S., Pereira, W., Cilli, E., Sale, C., Gualano, B., Otaduy, M., \& Artioli, G. (2020). Magnetic resonance spectroscopy as a non-invasive method to quantify muscle carnosine in humans: a comprehensive validity assessment. Scientific Reports, 10(1), 4908.

Danaher, J., Gerber, T., Wellard, R. M., \& Stathis, C. G. (2014). The effect of $\beta$-alanine and $\mathrm{NaHCO} 3$ co-ingestion on buffering capacity and exercise performance with high-intensity exercise in healthy males. European Journal of Applied Physiology, 114(8), 1715-1724.

Dankel, S. J., Bell, Z. W., Spitz, R. W., Wong, V., Viana, R. B., Chatakondi, R. N., Buckner, S. L., Jessee, M. B., Mattocks, K. T., Mouser, J. G., Abe, T., \& Loenneke, J. P. (2020). Assessing differential responders and mean changes in muscle size, strength, and the crossover effect to 2 distinct resistance training protocols. Applied Physiology, Nutrition, and Metabolism, 45(5), 463-470.

Derave, W., Ozdemir, M. S., Harris, R. C., Pottier, A., Reyngoudt, H., Koppo, K., Wise, J. a, \& Achten, E. (2007). Beta-Alanine supplementation augments muscle carnosine content and attenuates fatigue during repeated isokinetic contraction bouts in trained sprinters. Journal of Applied Physiology (Bethesda, Md. : 1985), 103(5), 1736-1743.

Dolan, E., Saunders, B., Harris, R., Bicudo, E., Bishop, D., Sale, C., \& Gualano, B. (2019). Comparative physiology investigations support a role for histidinecontaining dipeptides in intracellular acid-base regulation of skeletal muscle. Comparative Biochemistry and Physiology Part A: Molecular and Integrative Physiology, 234, 77-86.

Gelman, A., Carlin, J. B., Stern, H. S., Dunson, D. B., Vehtari, A., \& Rubin, D. B. (2013). Bayesian Data Analysis (3rd ed.). CRC Press. 
Guyatt, G., Oxman, A., Vist, G., Kunz, R., Falck-Ytter, Y., Alonso-Coello, P., Schunemann, H., \& GRADE working group. (2008). GRADE: an emerging consensus on rating quality of evidence and strength of recommendations. British Medical Journal (Clinical Research Ed), 336(7650), 924-926.

Harris, R., Tallon, M., Dunnett, M., Boobis, L., Coakley, J., Kim, H., Fallowfield, J. L., Hill, C., Sale, C., \& Wise, J. (2006). The absorption of orally supplied beta-alanine and its effect on muscle carnosine synthesis in human vastus lateralis. Amino Acids, 30(3), 279-289.

Hecksteden, A., Kraushaar, J., Scharhag-Rosenberger, F., Theisen, D., Senn, S., \& Meyer, T. (2015). Individual response to exercise training - a statistical perspective. Journal of Applied Physiology, 118(12), 1450-1459.

Hill, C., Harris, R., Kim, H., Harris, B., Sale, C., Boobis, L., Kim, C., \& Wise, J. (2007). Influence of $\beta$-alanine supplementation on skeletal muscle carnosine concentrations and high intensity cycling capacity. Amino Acids, 32(2), 225-233.

Islam, H., \& Gurd, B. (2020). Exercise response variability: Random error or true differences in exercise response? Experimental Physiology, 105(12), 2022-2024.

Jones, R. L., Barnett, C. T., Davidson, J., Maritza, B., Fraser, W. D., Harris, R., \& Sale, C. (2017). $\beta$-alanine supplementation improves in-vivo fresh and fatigued skeletal muscle relaxation speed. European Journal of Applied Physiology, 117(5), 867879.

Kelley, G., \& Kelley, K. (2019). Systematic reviews and meta-analysis in nutrition research. British Journal of Nutrition, 122, 1279-1294.

Mann, T. N., Lamberts, R. P., \& Lambert, M. I. (2014). High Responders and Low Responders: Factors Associated with Individual Variation in Response to 
Standardized Training. Sports Medicine, 44(8), 1113-1124.

Marticorena, F., Carvalho, A., de Oliveira, L., Dolan, E., Gualano, B., Swinton, P., \& Saunders, B. (2021). Nonplacebo controls to determine the magnitude of ergogenic interventions: A systematic review and meta-analysis. Medicine and Science in Sports and Exercise, 10.1249/MSS.0000000002635.

McGuinness, L. A., \& Higgins, J. P. T. (2021). Risk-of-bias VISualization (robvis): An $\mathrm{R}$ package and Shiny web app for visualizing risk-of-bias assessments. Research Synthesis Methods, 12(1), 55-61.

Moher, D., Liberati, A., Tetzlaff, J., Altman, D. G., Altman, D., Antes, G., Atkins, D., Barbour, V., Barrowman, N., Berlin, J. A., Clark, J., Clarke, M., Cook, D., D’Amico, R., Deeks, J. J., Devereaux, P. J., Dickersin, K., Egger, M., Ernst, E., ... Tugwell, P. (2009). Preferred reporting items for systematic reviews and metaanalyses: The PRISMA statement. PLoS Medicine, 6(7).

Morris, S., \& DeShon, R. (2002). Combining effect size estimates in meta-analysis with repeated measures and independent-group designs. Psychological Methods, 7(1), $105-125$.

Patel, K. A., Farias de Oliveira, L., Sale, C., \& James, R. M. (2021). The effect of $\beta$ alanine supplementation on high intensity cycling capacity in normoxia and hypoxia. Journal of Sports Sciences, 1-7.

Rezende, N. S., Swinton, P., de Oliveira, L. F., da Silva, R. P., da Eira Silva, V., Nemezio, K., Yamaguchi, G., Artioli, G. G., Gualano, B., Saunders, B., \& Dolan, E. (2020). The Muscle Carnosine Response to Beta-Alanine Supplementation: A Systematic Review With Bayesian Individual and Aggregate Data E-Max Model and Meta-Analysis. Frontiers in Physiology, 11. 
Sale, C., Hill, C. A., Ponte, J., \& Harris, R. C. (2012). B-Alanine Supplementation Improves Isometric Endurance of the Knee Extensor Muscles. Journal of the International Society of Sports Nutrition, 9(1), 26. https://doi.org/10.1186/1550$2783-9-26$

Sale, C., Saunders, B., Hudson, S., Wise, J. A., Harris, R. C., \& Sunderland, C. D. (2011). Effect of $\beta$-Alanine plus Sodium Bicarbonate on High-Intensity Cycling Capacity. Medicine \& Science in Sports \& Exercise, 43(10), 1.

Saunders, B., de Salles Painelli, V., de Oliveira, L. F., da Eira Silva, V., da Silva, R. P., Riani, L., Franchi, M., Gonçalves, L. D. S., Harris, R. C., Roschel, H., Artioli, G. G., Sale, C., \& Gualano, B. (2017). Twenty-four Weeks of $\beta$-Alanine Supplementation on Carnosine Content, Related Genes, and Exercise. Medicine and Science in Sports and Exercise, 49(5), 896-906.

Saunders, B., Elliott-Sale, K., Artioli, G. G., Swinton, P. A., Dolan, E., Roschel, H., Sale, C., \& Gualano, B. (2017). $\beta$-alanine supplementation to improve exercise capacity and performance: a systematic review and meta-analysis. British Journal of Sports Medicine, 51(8), 658-669.

Saunders, B., Sale, C., Harris, R. C., Morris, J. G., \& Sunderland, C. (2013). Reliability of a high-intensity cycling capacity test. Journal of Science and Medicine in Sport, 16(3), 286-289.

Senn, S. (2004). Individual response to treatment: is it a valid assumption? British Medical Journal, 329(7472), 966-968.

Sterne, J. A. C., Savović, J., Page, M. J., Elbers, R. G., Blencowe, N. S., Boutron, I., Cates, C. J., Cheng, H.-Y., Corbett, M. S., Eldridge, S. M., Emberson, J. R., Hernán, M. A., Hopewell, S., Hróbjartsson, A., Junqueira, D. R., Jüni, P., Kirkham, J. J., Lasserson, T., Li, T., ... Higgins, J. P. T. (2019). RoB 2: a revised 
tool for assessing risk of bias in randomised trials. BMJ, 14898.

Swinton, P. A., Hemingway, B. S., Saunders, B., Gualano, B., \& Dolan, E. (2018). A Statistical Framework to Interpret Individual Response to Intervention: Paving the Way for Personalized Nutrition and Exercise Prescription. Frontiers in Nutrition, 5 (May), 41.

Trexler, E. T., Smith-Ryan, A. E., Stout, J. R., Hoffman, J. R., Wilborn, C. D., Sale, C., Kreider, R. B., Jäger, R., Earnest, C. P., Bannock, L., Campbell, B., Kalman, D., Ziegenfuss, T. N., \& Antonio, J. (2015). International society of sports nutrition position stand: Beta-Alanine. Journal of the International Society of Sports Nutrition, 12(1), 30.

Watson, J. A., Ryan, C. G., Atkinson, G., Williamson, P., Ellington, D., Whittle, R., Dixon, J., \& Martin, D. J. (2021). Inter-Individual Differences in the Responses to Pain Neuroscience Education in Adults With Chronic Musculoskeletal Pain: A Systematic Review and Meta-Analysis of Randomized Controlled Trials. The Journal of Pain, 22(1), 9-20.

Williamson, P. J., Atkinson, G., \& Batterham, A. M. (2018). Inter-individual differences in weight change following exercise interventions: a systematic review and meta-analysis of randomized controlled trials. Obesity Reviews, 19(7), 960975.

Williamson, Philip J., Atkinson, G., \& Batterham, A. M. (2017). Inter-Individual Responses of Maximal Oxygen Uptake to Exercise Training: A Critical Review. Sports Medicine, 47(8), 1501-1513.

Yamaguchi, G. C., Nemezio, K., Schulz, M. L., Natali, J., Cesar, J. E., Riani, L. A., de Souza Gonçalves, L., Möller, G. B., Sale, C., de Medeiros, M. H. G., Gualano, B., \& Artioli, G. G. (2020). Kinetics of Muscle Carnosine Decay after $\beta$-alanine 
Supplementation. Medicine \& Science in Sports \& Exercise, Published Ahead of Print. 
Tables

Table 1: Inclusion and exclusion criteria according to PICOS

Population Healthy participants of any age or physical activity level.

Intervention Studies investigating the effects of chronic BA supplementation $(\geq 4$ weeks) on exercise test performance.

Comparator Change in the BA group versus placebo.

Outcomes The primary outcome of interest was change in TTE in the $\mathrm{CCT}_{110 \%}$ test, along with individual response variation. Changes in MCarn content were considered a secondary outcome, along with the proposed moderator analyses described in the analysis section.

Study design Randomized, double-blinded, placebo-controlled intervention studies. 
1 Table 2: Characteristics of Included Studies

\begin{tabular}{|c|c|c|c|}
\hline Author (date) & Study design & Population & Dosing Protocol \\
\hline Hill et al. (2007) & $\begin{array}{l}\text { Double blinded RCT, with tests conducted } \\
\text { after } 0,4,8 \text { and } 10 \text { weeks of } \\
\text { supplementation. }\end{array}$ & $\begin{array}{l}\text { Healthy, physically active males (BA } n \\
=13 ; \text { PLA } n=12) .\end{array}$ & $\begin{array}{l}\text { BA: Week 1: } 4 \mathrm{~g} \cdot \text { day }^{-1} \cdot \text { Week } 2: 4.8 \mathrm{~g} \cdot \text { day }^{-1} \text {. Week } 3: 5.6 \\
\mathrm{~g} \cdot \text { day }^{-1} \text {. Week } 4: 6.4 \mathrm{~g} \cdot \text { day }^{-1} \text {. Dose maintained until } \\
\text { week } 10 \text { for } 8 \text { participants }(8 \times 800 \mathrm{mg} \text { doses }) \text { (total dose } \\
=145.6 \mathrm{~g}) \text {. PLA: maltodextrin. }\end{array}$ \\
\hline Sale et al. (2011) & $\begin{array}{l}\text { Double blinded RCT, with tests conducted } \\
\text { after } 0 \text { and } 4 \text { weeks of supplementation. }\end{array}$ & $\begin{array}{l}\text { Healthy, physically active males (BA } n \\
=10 ; \text { PLA } n=10) .\end{array}$ & $\begin{array}{l}\text { BA: } 6.4 \mathrm{~g} \cdot \mathrm{day}^{-1}(4 \times 1600 \mathrm{mg}) \text { for } 4 \text { weeks (total dose }= \\
179.2 \mathrm{~g}) . \text { PLA: maltodextrin. }\end{array}$ \\
\hline Danaher et al. (2014) & $\begin{array}{l}\text { Double blinded, within subject cross-over, } \\
\text { RCT, with test sessions separated by a } 12 \text { - } \\
\text { week washout. }\end{array}$ & Healthy, physically active males $(n=8)$. & $\begin{array}{l}\text { BA: } 4.8 \mathrm{~g} \cdot \text { day }^{-1}(6 \times 800 \mathrm{mg}) \text { for } 4 \text { weeks, then } 6.4 \mathrm{~g} \cdot \text { day }^{-} \\
\left.{ }^{1}(8 \times 800 \mathrm{mg}) \text { for } 2 \text { weeks (total dose }=224 \mathrm{~g}\right) \text {. PLA: } \\
\text { calcium carbonate. }\end{array}$ \\
\hline Saunders et al. (2017) & $\begin{array}{l}\text { Double blinded RCT, with tests conducted } \\
\text { after } 0,4,8,12,16,20 \text {, and } 24 \text { weeks of } \\
\text { supplementation. }\end{array}$ & $\begin{array}{l}\text { Healthy, physically active males (BA } n \\
=15 ; \text { PLA } n=9) \text {. }\end{array}$ & $\begin{array}{l}\text { BA: } 6.4 \mathrm{~g} \cdot \text { day }^{-1} \text { of BA }(4 \times 1600 \mathrm{mg}) \text { for } 24 \text { weeks (total } \\
\text { dose }=1075.2 \mathrm{~g}) . \text { PLA: maltodextrin. }\end{array}$ \\
\hline Yamaguchi et al. (2020) & $\begin{array}{l}\text { Double blinded RCT, with tests conducted } \\
\text { after } 0 \text { and } 8 \text { weeks of supplementation. }\end{array}$ & $\begin{array}{l}\text { Healthy, physically active, omnivorous } \\
\text { males (BA } n=11 \text {; PLA } n=4)\end{array}$ & $\begin{array}{l}\text { BA: } 6.4 \mathrm{~g} \cdot \text { day }^{-1}(4 \times 1600 \mathrm{mg}) \text { for } 8 \text { weeks (total dose }= \\
358.4 \mathrm{~g}) . \text { PLA: maltodextrin. }\end{array}$ \\
\hline Patel et al. (2021) & $\begin{array}{l}\text { Double blinded RCT, with tests conducted } \\
\text { after } 0 \text { and } 4 \text { weeks of supplementation. }\end{array}$ & $\begin{array}{l}\text { Healthy, physically active, omnivorous } \\
\text { males (BA } n=10 ; \text { PLA } n=9 \text { ) }\end{array}$ & $\begin{array}{l}\text { BA: } 6.4 \mathrm{~g} \cdot \mathrm{day}^{-1}(4 \times 1600 \mathrm{mg}) \text { for } 4 \text { weeks (total dose }= \\
179.2 \mathrm{~g}) . \text { PLA: celluloses plus excipients. }\end{array}$ \\
\hline
\end{tabular}


3 Table 3: GRADE Quality assessment and summary of findings

\begin{tabular}{|c|c|c|c|c|c|c|c|c|c|}
\hline \multicolumn{6}{|l|}{ Quality assessment } & \multirow{2}{*}{\multicolumn{2}{|c|}{$\begin{array}{l}\text { Summary of findings } \\
\text { Number of participants }\end{array}$}} & \multirow[b]{3}{*}{ Effect size $(95 \% \mathrm{CrI})$} & \multirow[b]{3}{*}{ Quality } \\
\hline & & & & & & & & & \\
\hline Outcome (No. of studies) & ROB & Consistency & Precision & Publication Bias & Upgrade & BA & PLA & & \\
\hline TTE main effect of BA (AD) (5) & $\oplus \oplus \oplus \oplus$ & $\oplus \oplus \oplus \oplus$ & $\oplus \oplus \oplus \oplus$ & $\oplus \oplus \oplus \oplus$ & $\oplus \oplus \oplus \oplus$ & 59 & 44 & $11.9(6.3-16.5) \mathrm{s}$ & $\begin{array}{c}\oplus \oplus \oplus \oplus \\
\text { High }\end{array}$ \\
\hline TTE main effect of BA (IPD) (4) & $\oplus \oplus \oplus \oplus$ & $\oplus \oplus \oplus \oplus$ & $\oplus \oplus \oplus \oplus$ & $\oplus \oplus \oplus \oplus$ & $\oplus \oplus \oplus \oplus$ & 46 & 32 & $7.7(1.3-14.3) \mathrm{s}$ & $\begin{array}{c}\oplus \oplus \oplus \oplus \\
\text { High }\end{array}$ \\
\hline $\begin{array}{l}\text { TTE influence of total BA } \\
\text { consumption }(500 \mathrm{~g})(2)\end{array}$ & $\bigoplus \oplus \oplus \oplus$ & $\oplus \oplus \oplus \oplus$ & $\oplus \oplus \oplus \bigcirc$ & $\bigoplus \oplus \oplus \bigcirc$ & $\oplus \oplus \oplus \bigcirc$ & 26 & 13 & $7.4(0.8-13.9) \mathrm{s}$ & $\begin{array}{c}\oplus \oplus \oplus \bigcirc \\
\text { Moderate }\end{array}$ \\
\hline TTE response variation $\left(\sigma_{I R}\right)(\mathbf{4})$ & $\bigoplus \oplus \oplus \oplus$ & $\oplus \oplus \oplus \oplus$ & $\oplus \oplus \oplus \oplus$ & $\oplus \oplus \oplus \oplus$ & $\bigoplus \oplus \oplus \oplus$ & 46 & 32 & - & $\begin{array}{c}\oplus \oplus \oplus \oplus \\
\text { High }\end{array}$ \\
\hline MCarn main effect of BA (AD) (4) & $\oplus \oplus \oplus \oplus$ & $\oplus \oplus \oplus \oplus$ & $\oplus \oplus \oplus \bigcirc$ & $\oplus \oplus \oplus \bigcirc$ & $\oplus \oplus \oplus \oplus$ & 40 & 27 & $\begin{array}{l}13.7(7.7-19.6) \\
\mathrm{mmol}^{\prime} \cdot \mathrm{kgDM}^{-1}\end{array}$ & $\begin{array}{c}\oplus \oplus \oplus \oplus \\
\text { High }\end{array}$ \\
\hline MCarn main effect of BA (IPD) (2) & $\oplus \oplus \oplus \oplus$ & $\oplus \oplus \oplus \oplus$ & $\oplus \oplus \oplus \bigcirc$ & $\oplus \oplus \oplus \bigcirc$ & $\oplus \oplus \oplus \oplus$ & 26 & 13 & $\begin{array}{c}18.1(14.5-21.9) \\
\mathrm{mmol} \cdot \mathrm{kgDM}^{-1}\end{array}$ & $\begin{array}{c}\oplus \oplus \oplus \oplus \\
\text { High }\end{array}$ \\
\hline $\begin{array}{l}\text { MCarn influence of total BA } \\
\text { consumption }(500 \mathrm{~g})(2)\end{array}$ & $\oplus \oplus \oplus \oplus$ & $\oplus \oplus \oplus \oplus$ & $\oplus \oplus \oplus \bigcirc$ & $\oplus \oplus \oplus \bigcirc$ & $\bigoplus \oplus \oplus \oplus$ & 26 & 13 & $\begin{array}{l}15.1(10.7-19.5) \\
\mathrm{mmol} \cdot \mathrm{kgDM}^{-1}\end{array}$ & $\begin{array}{c}\oplus \oplus \oplus \oplus \\
\text { High }\end{array}$ \\
\hline MCarn response variation $\left(\sigma_{I R}\right)$ & $\oplus \oplus \oplus \oplus$ & $\oplus \oplus \oplus \oplus$ & $\oplus \oplus \oplus \bigcirc$ & $\oplus \oplus \oplus \bigcirc$ & $\oplus \oplus \oplus \oplus$ & 26 & 13 & $\begin{array}{c}5.8(4.2-7.4) \\
\mathrm{mmol} \cdot \mathrm{kgDM}^{-1}\end{array}$ & $\begin{array}{c}\oplus \oplus \oplus \oplus \\
\text { High }\end{array}$ \\
\hline
\end{tabular}

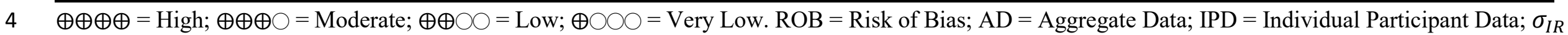

$5=$ Intervention Response Standard Deviation. 


\section{Figure 1}

(a)

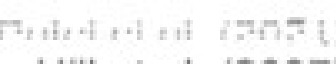

Hili et al. (2007)

- ....

to - voural. (2011)

Saunders et al. (2017b)

Yamaguchi et al. (2021)

Patel et al. (2021)

Pooled

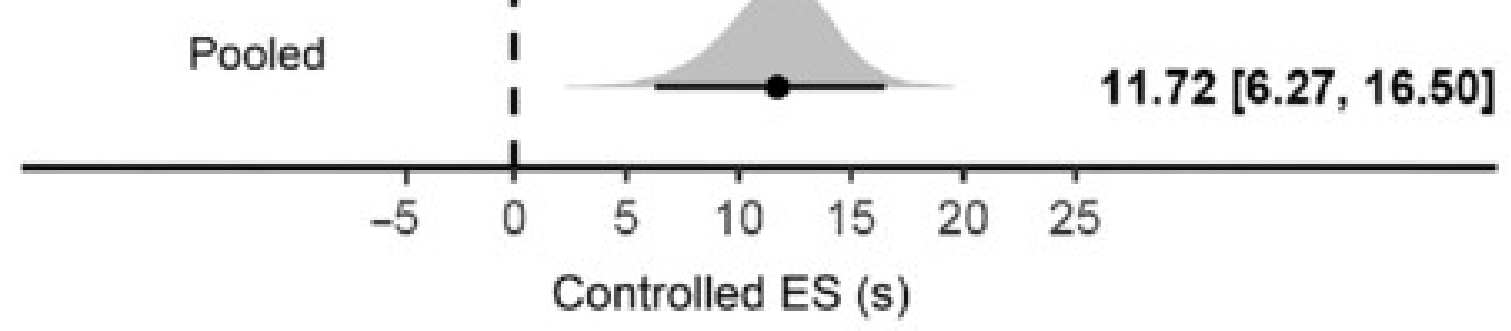

$12.88[7.90,17.47]$

$12.22[4.26,20.49]$

$11.76[6.71,16.39]$

$10.82[0.93,17.90]$

$10.75[0.70,17.74]$
Controlled ES (s)

(b)

Sale et al. (204t)

Saunders et al. (2017b)

Yamaguchi et al. (2021)

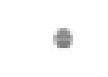

$-\quad-m-\infty$

- $1=$ - - - ramaquent et -10 을

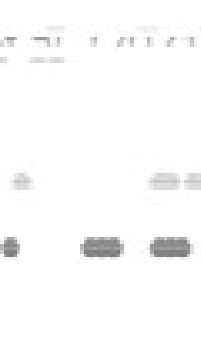

Patel et al. (2021)

Pooled

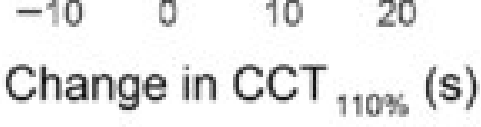

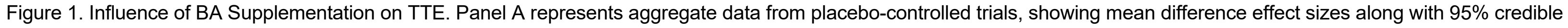

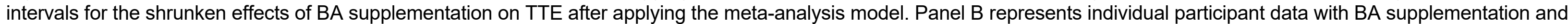
placebo means along with $95 \%$ credible intervals. 
Figure 2

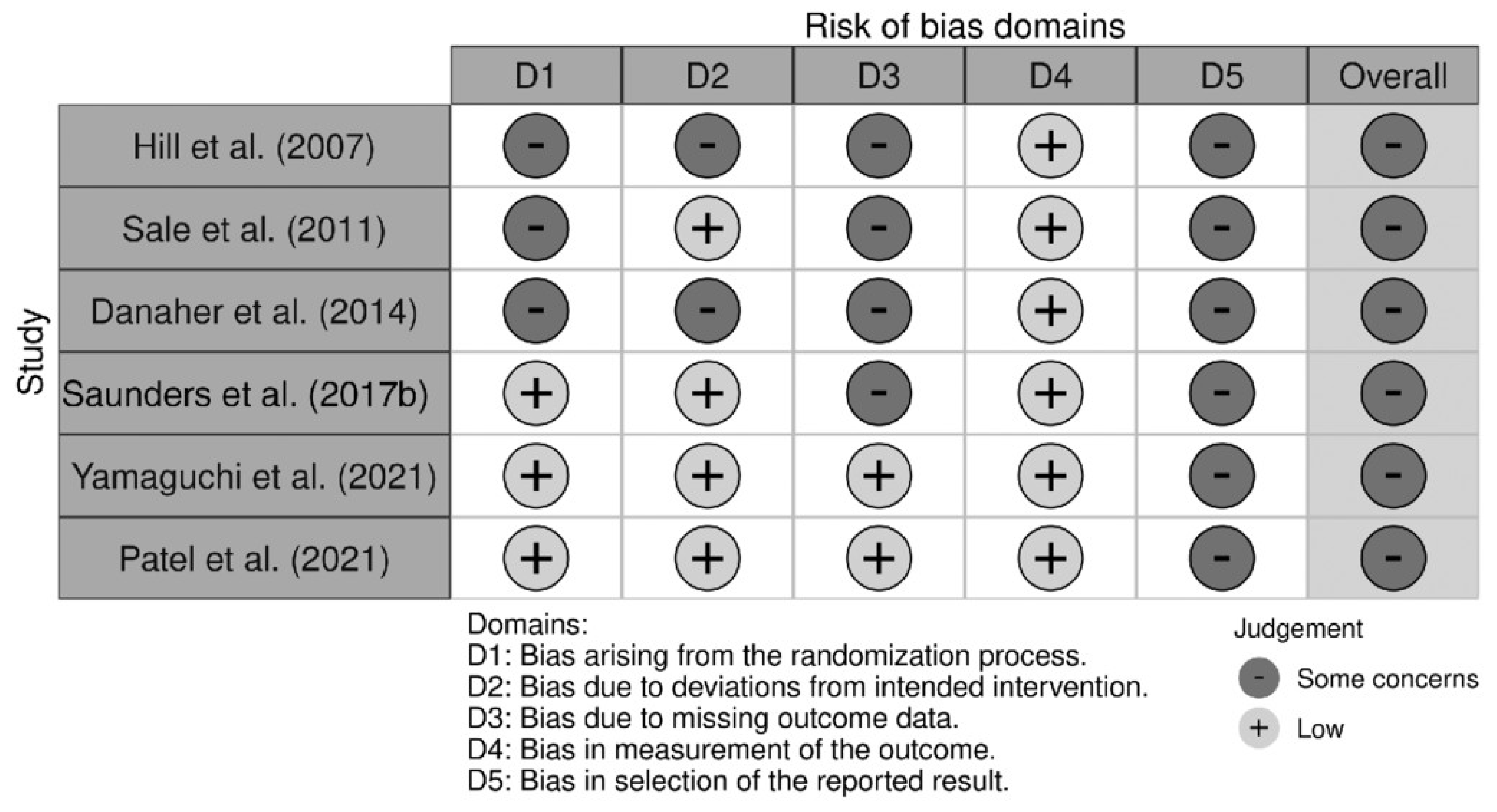

